\title{
Bowel Preparation for Colonoscopy in Children: 1 Day PEG-3350 with Bisacodyl versus 3 Day Sennosides
}

\author{
Engin Tutar Nevzat Aykut Bayrak Burcu Volkan Deniz Ertem \\ Division of Pediatric Gastroenterology, Hepatology and Nutrition, Marmara University School of Medicine, \\ Istanbul, Turkey
}

\section{Keywords}

Children · Colonoscopy · Bowel preparation · Polyethylene glycol $3350 \cdot$ Bisacodyl · Sennosides

\footnotetext{
Abstract

Background and Objectives: Bowel preparation (BP) for colonoscopy is a challenging procedure in children and different regimens have been used for this purpose. Polyethylene glycol (PEG) is the most preferred agent in recent years. The primary aim of this study was to evaluate the efficacy of 1-day PEG-3350 with bisacodyl (PEG-B) and comparing it with 3-day sennosides A+B. Method: In this prospective, randomized, and single-blinded study, children aged 2-18years were included in the PEG-B group for 1 day or in Senna group for 3 days. The effectiveness of BP was assessed according to the Ottawa and Boston BP scales, compliance and adverse effects were also recorded. Pre- and post-preparation biochemistry were obtained for investigation of safety of
}

both regimens. Results: Successful BP was observed in $88.3 \%$ ( $n=53 / 60)$ of PEG-B and $86 \%(n=55 / 64)$ of Senna groups according to Boston scale, and it was $85 \%(n=51 / 60)$ and $84.4 \%$ ( $n=54 / 64)$, respectively, according to Ottawa scale. The cecal intubation rate was $96.7 \%(n=58 / 60)$ in the PEG-B group and $93.8 \%(n=60 / 64)$ in the Senna group. Ease of administration and disturbance in regular daily activities was better in the PEG-B group $(p<0.05)$. There was no major adverse event and biochemical abnormality in both groups. The correlation between Ottawa and Boston scales was found to be excellent $\left(r^{2}=-0.954, p<0.01\right)$. Conclusions: The efficacy, safety, and adverse effect profile of 1-day BP with PEG-B regimen was found to be similar to 3-day sennosides regimen, however, the PEG-B regimen had advantages such as short duration, ease of administration, and better patient comfort. Also, high correlation rate between the Boston and Ottawa scales in pediatric patients was remarkable.

(c) 2019 S. Karger AG, Basel

\section{KARGER}

(c) 2019 S. Karger AG, Basel

E-Mail karger@karger.com

www.karger.com/ddi
Prof. Dr. Deniz Ertem Chief

Division of Pediatric Gastroenterology

Marmara University School of Medicine Başıüyük

Campus Maltepe Başıbüyük Yolu Sok. Number 9/1, TR-34854 Istanbul (Turkey)

E-Mail denizertem@marmara.edu.tr 


\section{Introduction}

Colonoscopy is an important diagnostic and therapeutic tool for various gastrointestinal disorders [1]. A successful colonoscopy is affected by multiple factors, and adequate bowel cleansing is one of the most important one [2]. Suboptimal bowel preparation (BP) can occur in up to one-third of the colonoscopies [1,3]. Insufficient $\mathrm{BP}$ may result in poor visualization of the colon, missing the lesions, prolonged procedure time, unsuccessful therapeutic intervention, potential risk for complications, and financial burden of a repeat procedure [1]. An adequate $\mathrm{BP}$ should be palatable, meet the requirements for cleansing quality in low volumes, short lasting, and not associated with significant adverse events $[1,2]$. There is no standard pediatric BP regimen. However, short course regimens, consisting of lower volumes are becoming more acceptable by pediatric gastroenterologists.

Bowel cleansing for colonoscopy is a challenging procedure in children as it usually comprises dietary restriction, consumption of large amount of fluids for several days, and ingestion of unpalatable oral cleansing agents. Most of the cleansing regimens contain an osmotic (polyethylene glycol [PEG], lactulose) or a stimulant laxative (bisacodyl, senna, sodium picosulfate) or a combination of both agents. However, evidence for laxative stimulants alone is inconclusive, and frequently used in combination with osmotic laxatives in cleansing protocols [1]. PEG3350 has been successfully used in long-term treatment of chronic constipation, and it has become the most popular osmotic laxative included into the bowel cleansing regimens. The duration of PEG-3350 regimens range between 1 and 4 days [4-10]. However, it has been shown that the longer the duration of the regimen, the lower the compliance and the longer the abstinence from school or work. Therefore, short course regimens are particularly more acceptable for pediatric patients.

The primary aim of this trial was to compare the efficacy of 1-day PEG-3350 regimen with 3-day Senna regimen in children. The secondary aim was to compare the compliance, acceptance, safety, and tolerability of the 2 protocols.

\section{Materials and Method}

\section{Patients and Study Design}

This randomized, controlled, and single-blinded trial was conducted between April 2017 and May 2018. This study was approved by the local ethical board of Marmara University School of Medicine. Patients aged 2-18 years, scheduled to have an outpatient colonoscopy for various indications were enrolled for the study. Written informed consent was obtained from parents, and a written assent was also obtained from patients older than 14 years. The exclusion criteria for the study include allergy to laxatives, emergency colonoscopy, previous bowel surgery, and the presence of any metabolic, cardiac or renal disease. Patients having a history of chronic constipation were also excluded. Eligible patients were randomly chosen per protocol by block randomization. The instructions for BP were given by the same investigator. All colonoscopies were performed under deep intravenous sedation with midazolam $(0.05-0.1 \mathrm{mg} / \mathrm{kg})$ and propofol (initial induction dose, $1 \mathrm{mg} / \mathrm{kg}$; $0.5 \mathrm{mg} / \mathrm{kg}$ additional bolus doses every 3-5 min), administered by an anesthesiologist. The colonoscopist who was blind to the BP protocol, assessed the efficiency of bowel cleansing.

\section{Bowel Preparation}

The Senna group received sennosides A + B calcium (X-M solution) at a dose of $3 \mathrm{mg} / \mathrm{kg} /$ day (max. $150 \mathrm{mg} /$ day) in 2 divided doses for 3 days. Patients were instructed to consume full liquid diet on the first 2 days, and clear liquid diet 1 day before the colonoscopy. The minimum daily liquid volume the patient should consume was calculated according to daily maintenance fluid calculation formula per weight: for children up to $10 \mathrm{~kg} 100 \mathrm{~mL} / \mathrm{kg}$, for children 11-20 kg 1,000 mL $+50 \mathrm{~mL} / \mathrm{kg}$ for every $\mathrm{kg}$ over 10 and for children $>20 \mathrm{~kg}, 1,500 \mathrm{~mL}+20 \mathrm{~mL} / \mathrm{kg}$ for every kg over 20 . Instructed fluid volume was $10 \%$ more than the calculated daily liquid volume up to a maximum of 2,000 $\mathrm{mL}$ daily, to keep the patient adequately hydrated. However, patients were free to consume more fluid if they desire.

The PEG-B group received PEG-3350 (Movicol $13.8 \mathrm{~g}$ sachet) $2 \mathrm{~g} / \mathrm{kg} /$ day (max. $55.2 \mathrm{~g} /$ day) in instructed fluid volume amount of water or juice 1 day before the colonoscopy between 8 a.m. and 14 p.m. Bisacodyl dose was $5 \mathrm{mg} /$ day in children less than $30 \mathrm{~kg}$ once in the morning and $10 \mathrm{mg} /$ day for over $30 \mathrm{~kg}$ equally divided into 2 doses. Patients were instructed to consume only clear liquid diet before the day of colonoscopy.

All patients in the study group were instructed to follow nil per os 6-8 h before the colonoscopy.

\section{Assessment of Bowel Preparation}

All the colonoscopies were performed by an experienced pediatric gastroenterologist. The colonoscopist was blind to the bowel cleansing protocol, and assessed the quality of the preparation by both Ottawa bowel preparation scale (OBPS) and Boston bowel preparation scale (BBPS) $[11,12]$. Both scales scored the right, transverse, and the left segments of the colon separately. In BBPS, the efficacy of cleansing was scored between 0 and $3(0=$ inadequate cleansing, 3 = excellent cleansing) for each colonic segment, and a total BBPS score of 6-7 points was accepted as good, and 8-9 as excellent preparation [13]. In OBPS, the cleanliness of 3 colonic segments was scored into 5 categories $(0=$ excellent cleansing, $4=$ inadequate cleansing), and the amount of fluid accumulation in the colonic lumen, which may impair the colonic visualization, was also considered in this scale. The accumulated total colonic fluid was graded (between 0 and 2) and added to the sum of segmental colonic scores. Regimens used in this study were compared by using both the scales, and a total OBPS score between 3 and 5 was considered as good and $0-2$ as excellent preparation. When it was not possible to reach the cecum because of unsuccessful BP, the lowest score was given to the unobserved segments of the colon. 


\section{Patient Questionnaires and Safety}

Before the initiation of bowel cleansing, patients were asked to note their stool frequency and consistency daily for 1 week, and throughout the BP according to the modified Bristol stool form scale for children that maximum 5 points represents watery stool description [14]. In the Senna group, data belonging to the last cleansing day was considered statistically when compared with the PEG-B group.

Before the initiation of colonoscopy, patients and parents were asked to complete another questionnaire to assess compliance, ease, taste, and tolerability of the bowel cleansing protocol. Compliance was scored on a 3-point Likert scale according to the percentage of fluid consumed and medication used: excellent for $100 \%$, good for $>75 \%$ [15]. Ease was scored on a 5-point scale from " 1 = very easy" to " $5=$ very difficult" and taste was scored from " 1 = very well" to " $5=$ very bad". Tolerability was evaluated from records of adverse events from "none" to "severe". Willingness to repeat the same BP was also evaluated. Disturbance of regular daily activities were also questioned, and graded on a 4 -point scale from " $1=$ not at all" to " $4=$ very much".

Serum glucose, blood urea nitrogen, creatinine, sodium, potassium, chloride, calcium, magnesium, and phosphate levels were obtained twice from all participants within 15 days, before starting the $\mathrm{BP}$ and just before colonoscopy to monitor biochemical imbalances.

\section{Study End Points and Statistical Analysis}

The primary end point of our study was satisfactory colon preparation, defined as excellent/good by the BBPS and OBPS. Secondary end points were the ease, taste and tolerability of the BP, compliance, and willingness to repeat the procedure. Both bowelcleansing protocols were compared for primary and secondary end points.

Calculation of the sample size for this study was based on the primary end point, namely the comparison of the efficacy between 2 regimens. An efficacy of $80 \%$ has been reported in studies using PEG-3350, and the data for sennosides are scarce in children. Therefore, it was calculated that a sample size of 41 patients per group would be sufficient to detect a $20 \%$ difference between 2 groups, setting an alpha level at 0.05 and assuming a statistical power of $90 \%$.

The data were analyzed with a computer software program (IBM SPSS, version 20, IBM Corp., NY, USA). Continuous variables are presented as mean $\pm \mathrm{SD}$. Categorical variables are presented as numbers and percentages. The Student $t$ test was used to compare the clinical data of the groups. The repeated measure analysis of variance test was used to compare the changes between the groups. Correlations between 2 variables were evaluated by Pearson's correlation coefficient. The calculated $p$ values of less than 0.05 indicated statistical significance.

\section{Results}

\section{Study Sample and Patient Demographics}

The flowchart of the study is shown in Figure 1. During the study period, 151 colonoscopies were performed and 128 were eligible for the study. Patients were ran-

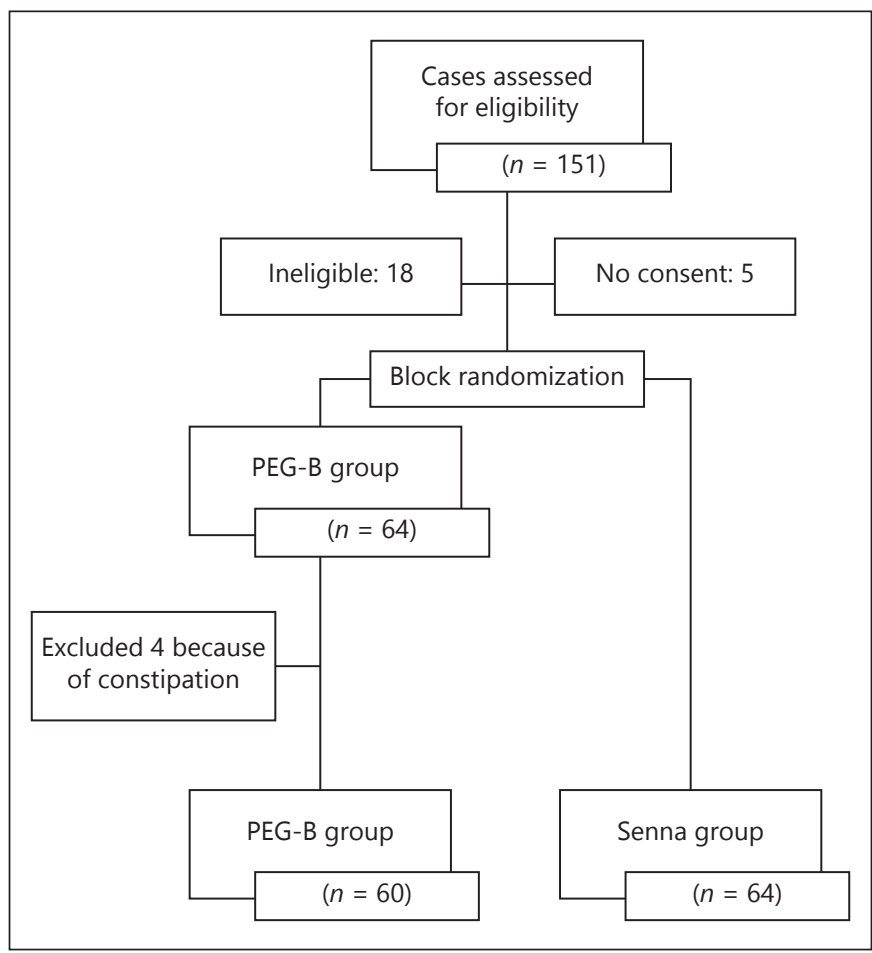

Fig. 1. Study flowchart.

domized into PEG-B and Senna groups by block randomization. A questionnaire was given to all patients to reveal the routine bowel habits of the group, and 4 patients who had serious constipation in PEG-B group were excluded. Ultimately, 60 children in PEG-B group and 64 children in Senna group completed the study; hence the statistical analysis was carried out according to per-protocol principle.

The mean age and sex distributions in PEG-B and Senna groups were $10.29 \pm 3.21$ vs. $10.11 \pm 4.25$ years and 53.3 vs. $51.6 \%$ girls, respectively $(p>0.05)$. The most common indication for colonoscopy was rectal bleeding in both groups. Stool frequency and consistency were not different statistically between the groups 1 week before the BP $(p>0.05$; Table 1$)$.

\section{Efficacy of BP}

All 124 patients had the BBPS and the OBPS scores at the end of the procedure. According to the BBPS, a good/excellent BP was achieved in $88.3 \%$ of the PEG-B and $86 \%$ of Senna groups, and according to the OBPS, it was 85 and $84.4 \%$, respectively. The efficacy of cleansing protocols was also evaluated by segmental colonic scores. There was no statistical difference in the efficacy of both protocols (Table 2). Full colonoscopy with visu- 
Table 1. Demographic characteristics, indications of colonoscopy and defecation frequency and consistency of the study group

\begin{tabular}{|c|c|c|c|}
\hline & $\begin{array}{l}\text { PEG-B group } \\
(n=60), n(\%)\end{array}$ & $\begin{array}{l}\text { Senna group } \\
(n=64), n(\%)\end{array}$ & $p$ value \\
\hline Age, years, mean \pm SD & $10.29 \pm 3.21$ & $10.11 \pm 4.25$ & ns \\
\hline Gender & & & ns \\
\hline Girl & $32(53.3)$ & $33(51.6)$ & \\
\hline Boy & $28(46.7)$ & $31(48.4)$ & \\
\hline Weight, kg, mean \pm SD & $28.34 \pm 14.71$ & $26.53 \pm 16.84$ & ns \\
\hline $\mathrm{BMI}, \mathrm{kg} / \mathrm{m}^{2}$, mean $\pm \mathrm{SD}$ & $20.78 \pm 2.31$ & $21.08 \pm 2.75$ & ns \\
\hline Indications for colonoscopy & & & ns \\
\hline Rectal bleeding & $35(58.4)$ & $39(60.9)$ & \\
\hline Chronic diarrhea & $14(23.3)$ & $16(25.0)$ & \\
\hline Abdominal pain & $9(15.0)$ & $7(10.9)$ & \\
\hline Other & $2(3.3)$ & $2(3.2)$ & \\
\hline \multicolumn{4}{|l|}{ Before bowel preparation } \\
\hline Stool frequency per day, mean \pm SD & $1.52 \pm 0.74$ & $1.7 \pm 0.74$ & ns \\
\hline Stool consistency score, mean \pm SD & $3.20 \pm 0.58$ & $3.34 \pm 0.69$ & ns \\
\hline \multicolumn{4}{|l|}{ The last day of bowel preparation } \\
\hline Stool frequency per day, mean \pm SD & $3.45 \pm 0.92$ & $3.44 \pm 0.83$ & ns \\
\hline Stool consistency per day, mean \pm SD & $4.85 \pm 0.40$ & $4.70 \pm 0.52$ & ns \\
\hline
\end{tabular}

BMI, body mass index; ns, non-significant.

alization of the cecum was not possible in 6 patients $(2$ in PEG-B group, 4 in Senna group) because of an inadequate BP. Cecal and ileal intubation rates were 96.7 and 95\% in the PEG-B group and 93.8 and $90.6 \%$ in the Senna group, respectively. The correlation between 2 bowel-cleansing scores in the evaluation of bowel cleansing was found to be excellent $\left(r^{2}=-0.954, p<0.01\right)$.

\section{Acceptability and Compliance}

In both groups, the compliance to the protocol was excellent/good in all patients. A significantly higher proportion of children in the PEG-B group compared to the Senna group rated the protocol as easy/very easy (78.3 vs. $43.7 \%$ respectively, $p<0.01$ ). Close to $30 \%$ of patients in the Senna group expressed difficulties in the consumption of the preparation, though, all of the patients completed the protocol. The palatability of the solutions was rated as well/very well by the majority of the study group, and there was no statistically significant difference between the regimens in this respect (Table 3).

\section{Tolerability and Safety}

There were only minor, self-limited adverse events among the participants, and none of the patients required an additional medication, hospitalization or intravenous rehydration. The most common complaints were abdominal distention/bloating, abdominal pain, and nausea in both study groups. These adverse events were not statistically different between the groups. Participants in the Senna group complained more about the disturbed daily activities (51.6 vs. $78.1 \%, p<0.02$ ). Patient willingness for repeating the same protocol if needed was higher in the PEG-B group compared to the Senna group (70 vs. $32.8 \%$ respectively, $p<0.01$; Table 3 ).

Serum biochemistry tests before the BP were within normal ranges in both groups. In addition, there was no statistically significant difference in serum biochemistry test results between the groups on the day of colonoscopy, but there was only mild deviation in serum sodium (PEG-B: 2 patients, Senna: 2 patients) and potassium (PEG-B: 2 patients, Senna: 1 patient) levels. However, there was no clinical sign of any electrolyte imbalance in these patients. Other biochemical tests, namely serum glucose and kidney function tests, were within normal range before and after the $\mathrm{BP}$.

\section{Discussion}

In this cohort, 1-day BP with PEG-3350 in combination with bisacodyl is effective, safe, and has high patient compliance and tolerability as a BP regimen in children. 
Table 2. Efficacy of BP between the PEG-B and Senna groups

\begin{tabular}{|c|c|c|c|}
\hline & $\begin{array}{l}\text { PEG-B group } \\
(n=60)\end{array}$ & $\begin{array}{l}\text { Senna group } \\
(n=64)\end{array}$ & $p$ value \\
\hline Total Boston BPS score, mean \pm SD & $7.58 \pm 1.75$ & $7.05 \pm 1.94$ & ns \\
\hline $\begin{array}{l}\text { Qualitative preparation rating } \\
\text { by BBPS, } n(\%)\end{array}$ & & & ns \\
\hline Excellent & $37(61.7)$ & $32(50)$ & \\
\hline Good & $16(26.7)$ & $23(35.9)$ & \\
\hline Poor & $5(8.3)$ & $6(9.4)$ & \\
\hline Inadequate & $2(3.3)$ & $3(4.7)$ & \\
\hline Successful BP (excellent + good) & $53(88.3)$ & $55(86)$ & ns \\
\hline $\begin{array}{l}\text { Boston BPS score per segment, } \\
\text { mean } \pm \text { SD }\end{array}$ & & & ns \\
\hline Left colon & $2.68 \pm 0.70$ & $2.67 \pm 0.77$ & \\
\hline Transvers colon & $2.58 \pm 0.61$ & $2.36 \pm 0.78$ & \\
\hline Right colon & $2.28 \pm 0.73$ & $2.02 \pm 0.67$ & \\
\hline $\begin{array}{l}\text { Total Ottawa BPS score, } \\
\text { mean } \pm \text { SD }\end{array}$ & $2.88 \pm 2.76$ & $3.52 \pm 3.01$ & ns \\
\hline $\begin{array}{l}\text { Qualitative preparation } \\
\text { rating by OBPS } n(\%)\end{array}$ & & & ns \\
\hline Excellent & $33(55)$ & $29(45.3)$ & \\
\hline Good & $18(30)$ & $25(39.1)$ & \\
\hline Poor & $7(11.7)$ & $7(10.9)$ & \\
\hline Inadequate & $2(3.3)$ & $3(4.7)$ & \\
\hline Successful BP (excellent + good), $n(\%)$ & $51(85)$ & $54(84.4)$ & ns \\
\hline $\begin{array}{l}\text { Ottawa BPS score per segment, } \\
\text { mean } \pm \text { SD }\end{array}$ & & & ns \\
\hline Left colon & $0.48 \pm 0.81$ & $0.80 \pm 0.99$ & \\
\hline Transvers colon & $0.63 \pm 0.88$ & $0.89 \pm 1.04$ & \\
\hline Right colon & $1.08 \pm 1.10$ & $1.27 \pm 0.94$ & \\
\hline Cecal intubation, $n(\%)$ & $58(96.7)$ & $60(93.8)$ & ns \\
\hline Ileal intubation, $n(\%)$ & $57(95)$ & $58(90.6)$ & ns \\
\hline
\end{tabular}

BPS, bowel preparation scale; BBPS, Boston bowel preparation scale; OBPS, Ottawa bowel preparation scale; ns, non-significant; BP, bowel preparation.

Although our conventional 3-day high dose sennosides regimen is equally effective, PEG-3350 combined with bisacodyl is superior in ease of application and willingness to repeat the preparation, in addition to minimal disturbance on regular daily activities.

Although there are a great number of bowel-cleansing protocols in use, there is not an ideal bowel-cleansing regimen, and there is still a search for an efficient, welltolerated, and short lasting regimen in children. PEG3350 based BP protocols have been very popular in the last 2 decades in children [1]. The efficacy of PEG for BP has been a subject of investigation for a long time, and satisfactory results varied between 70 and $95 \%$ in these studies $[16,17]$. However, the dose and duration of PEG used, the duration of clear liquid diet, and the medications added to PEG differ as well as the scales used to evaluate the efficacy of the BPs. In the present study, PEG-3350 combined with bisacodyl given 1 day was found to be successful in 88.3 and $85 \%$ of the children, according to the validated Boston and Ottawa scales, respectively.

The efficacy of BP regimen using PEG $1.5 \mathrm{~g} / \mathrm{kg} /$ day for 4 days were evaluated previously, and found to be effective in 91 and $89 \%$ of the patients $[4,18]$. Owing to the disadvantages of multiple day preparations such as poor compliance particularly in children, and disturbed daily activities, short-term PEG regimens were reported soon thereafter $[19,20]$. Lately, 1-day PEG-3350 regimens, administered in various doses and dissolved in maximum of $2 \mathrm{~L}$ fluid has become widely accepted by the pediatric gastroenterologists $[8,9]$. Two prospective pediatric studies evaluated the efficacy of 1-day high dose (max. $255 \mathrm{~g} /$ day) PEG regimen, and reported adequate cleansing in 75 and $100 \%$ of the patients $[7,9]$. But both studies lacked a validated method of evaluating BP. Similar high dose 1-day PEG regimen was assessed by Abbas et al. [8], and 77\% of the study population achieved effective preparation according to the Boston scale. However, the large fluid volume was stated as an obstacle of the regimen, and $6.5 \%$ of the patients were not able to complete it. More recently, it has been shown that lower doses of PEG (max. $51 \mathrm{~g} /$ day) given for 2 days demonstrated comparable efficiency of $88 \%$ by using Aronchick scale [20].

The combination of PEG with a stimulant laxative is another bowel cleansing regimen that was tried $[5,6$, $10,16,17]$. Two prospective pediatric studies investigated 2-day PEG-3350 combined with bisacodyl cleanout regimen $[5,6]$. In both studies, $2 \mathrm{~g} / \mathrm{kg}$ (max.136 g/ day) PEG was combined with bisacodyl to improve the efficacy of the regimen despite the shorter duration. Phatak et al. [5] reported excellent/good bowel cleansing in $92 \%$ of patients, whereas satisfactory cleansing was reported in only $73.6 \%$ of the children in the study published by Elitsur et al. [6]. The authors of the latter study explained that the lower efficacy rate of their regimen might be due to the use of a meticulous definition for adequate colon preparation. There are only a few studies evaluating the efficacy of the combination of 1-day PEG with a stimulant laxative for bowel cleansing $[10,16,17]$. In one of these, high dose PEG (max. $280 \mathrm{~g}$ ), in combination with bisacodyl, was found to be more effective than the same dose of PEG alone regimen with a success rate of 95 vs. $86 \%$, respectively [17]. Najafi et al. [16] tested the combination of PEG (2 g/kg/ day) with bisacodyl suppository, and reported a rather low efficacy rate of $70 \%$. Boston scale was used to assess the efficacy of the bowel cleansing methods in both studies. Another study performed by Sahn et al. [10] investigated 1-day high dose PEG-3350 (4 g/kg/day) 
Table 3. Compliance, ease, taste, and tolerability of the bowel preparation protocols

\begin{tabular}{|c|c|c|c|}
\hline & $\begin{array}{l}\text { PEG-B group } \\
(n=60)\end{array}$ & $\begin{array}{l}\text { Senna group } \\
(n=64)\end{array}$ & $p$ value \\
\hline Compliance, $n(\%)$ & & & ns \\
\hline Excellent & $51(85.0)$ & $53(82.8)$ & \\
\hline Good & $9(15)$ & $11(17.2)$ & \\
\hline Fair and poor & $0(0)$ & $0(0)$ & \\
\hline Ease, $n(\%)$ & & & $<0.01$ \\
\hline Very easy and easy & $47(78.3)$ & $28(43.7)$ & \\
\hline Neutral & $8(13.4)$ & $17(26.6)$ & \\
\hline Difficult and very difficult & $5(8.3)$ & $19(29.7)$ & \\
\hline Taste, $n(\%)$ & & & ns \\
\hline Very well and well & $50(83.3)$ & $52(81.3)$ & \\
\hline Neutral & $8(13.3)$ & $8(12.5)$ & \\
\hline Bad and very bad & $2(3.3)$ & $4(6.2)$ & \\
\hline \multicolumn{4}{|l|}{ Tolerability, $n(\%)$} \\
\hline Abdominal distention/bloating & $19(31.7)$ & $23(35.9)$ & ns \\
\hline Abdominal pain & $16(26.7)$ & $20(31.3)$ & ns \\
\hline Nausea and/or vomiting & $12(20)$ & $13(20.3)$ & ns \\
\hline Fecal incontinence & $3(5)$ & $2(3.1)$ & ns \\
\hline Sleep disturbance & $3(5)$ & $4(6.3)$ & ns \\
\hline $\begin{array}{l}\text { Disturbance in regular daily } \\
\text { activities, } n(\%)\end{array}$ & $31(51.7)$ & $50(78.1)$ & $<0.02$ \\
\hline $\begin{array}{l}\text { Willingness to repeat the } \\
\text { same preparation, } n(\%)\end{array}$ & $42(70)$ & $21(32.8)$ & $<0.01$ \\
\hline
\end{tabular}

combined with a single dose stimulant laxative in patients weighing more than $20 \mathrm{~kg}$, and the efficacy of the regimen was reported as $73.5 \%$. Aronchick scale was selected to evaluate the regimen in this study. In our 1-day regimen, a lower dose of PEG ( $1.5 \mathrm{~g} / \mathrm{kg} /$ day) was combined with bisacodyl to ameliorate the efficiency of the BP. This shorter regimen achieved excellent/good bowel cleansing in $85-88 \%$ of the children by using 2 different validated BP scales. In addition, there are some studies investigating the efficacy of bowel cleansing with short-term PEG and ascorbic acid combination, and reported satisfactory results $[13,21]$.

Bisacodyl and sennosides are preparations usually used in combination, rather than alone for the BP in children [1]. There are only a few reports investigating the effectiveness of stimulant laxatives in children. Studies in adults using high-dose sennosides alone showed highly efficient bowel cleansing with no major adverse events [22-26]. However, the results in pediatric age group differed from adult studies, and senna fared poorly against PEG [20]. A randomized controlled pediatric trial with sennosides $(130 \mathrm{mg} /$ day) combined with magnesium citrate for 2 days showed effective BP in $70 \%$ of the patients [3]. The efficacy of 3-day sennosides (150 $\mathrm{mg} /$ day) regimen, which is conventionally used in our tertiary center for the last decade, assessed the Boston and Ottawa scales separately, and demonstrated excellent or good efficiency in 86 and $84.4 \%$ of patients, respectively. The dose of sennosides used in our study is comparable, but our better cleansing result may arise from the longer duration of sennosoide regimen, namely 3 days. Besides, we also found a perfect correlation between the Boston and Ottawa scales in our cohort of children. Although it was not one of the aims of the study, using 2 scales for evaluating the regimens and a high correlation between them supports the reliability of our results.

Another criteria for the efficacy of the regimens were visualization of the cecum and ileal intubation, achieved in more than $95 \%$ of the patients in this study, with the success rates being slightly higher in the PEG-B group. These results are comparable to the rates reported with 1-day high-dose PEG-3350 regimens $[7,8]$ but much higher than the rate reported in the study conducted with a similar low dose of PEG-B [16].

The compliance in our study was found to be excellent or good in all patients, irrespective of the duration and the regimen used. Although more than $80 \%$ of the patients in both the groups found the taste of the regimens very well or well, significantly more patients in the PEG-B group found the BP easier compared to the Senna group (78.3 vs. $43.7 \%)$. Compatible with the rate of acceptance, the willingness to repeat the same preparation was higher in the PEG-B group (70 vs. $32.8 \%$ ). Studies rating the tolerability and acceptability of 1-day PEG regimen were reported as difficult or unacceptable in $29-31 \%$ of the population [10]. The most commonly reported complaint in these studies was the large volume of preparation required to ingest. This might necessitate inpatient $\mathrm{BP}$, and particularly addressed in the report of the NASPGHAN endoscopy and procedure committee report [1]. In the present study, disturbance in daily activities reported in $52 \%$ of children who received PEG-B was higher than in the study by Abbas et al. [8], which reported a rate of $24 \%$. The low rate of missing days from school in the latter study could be attributed to the starting of the regimen at the evening.

The most common adverse effects were abdominal pain followed by nausea, however, all of the subjects in the 2 regimen groups completed the $\mathrm{BP}$ and none required a nasogastric tube placement or hospitalization. The mild feature of adverse events in our study is compatible with previous studies which reported that PEG-based 
regimens do not cause any major adverse effects in children $[4,6-8,18,27]$.

Safety parameters, specifically serum electrolytes, were not evaluated in all pediatric studies concerning colonic preparation. Metabolic disturbances including hyperphosphatemia, hypocalcemia, hypo- or hypernatremia have been reported in association with oral sodium phosphate preparations in adults and children [1, $2,28,29]$. Biochemistry was evaluated only in a few prospective studies with 1-day PEG regimens. Some minor, clinically insignificant pre- and post-preparation metabolic changes were observed, especially in serum glucose level [7-10]. Sahn et al. [10] reported that changes in electrolyte level and renal function were not clinically significant at the post-preparation phase, but they underscored the importance of hypoglycemia, specifically in children younger than 7 years of age. In this study, minor electrolyte changes were observed in 4 patients in the PEG-B group, but resolved without any medical intervention.

The limitation of this study may be small sample size. This is a single tertiary center study; therefore, a well-experienced endoscopist performed all of the colonoscopies. The endoscopist was blind to the BP regimens; furthermore 2 different validated scales were used for the assessment of efficacy of the regimens. These are the strengths of the present study.

In conclusion, low-volume and low-dose PEG-3350 $\mathrm{BP}$ regimen in combination with bisacodyl for 1-day is as effective, safe, and tolerable as 3-day sennosides. However, PEG-B regimen should be preferred because of the ease of application and minimal disturbance on daily activities. Besides, the presence of a high correlation rate between the Boston and Ottawa scales in pediatric patients deserve attention.

\section{Acknowledgement}

None.

\section{Ethics Statement}

Written informed consent is obtained from all subjects' parents. The study protocol is approved by the local Ethical Committee of Marmara University School of Medicine.

\section{Disclosure Statement}

The authors declare that no financial or other conflict of interest exists in relation to the content of the article.

\section{Author Contributions}

E.T.: planned the study, wrote the initial draft and revised the manuscript. N.A.B.: planned the study, collected and analyzed the data, wrote the initial draft, and revised the manuscript. B.V.: collected the data and revised the manuscript. D.E.: planned the study and critically revised and finalized the manuscript.

\section{References}

1 Pall H, Zacur GM, Kramer RE, Lirio RA, Manfredi M, Shah M, et al. Bowel preparation for pediatric colonoscopy: report of the NASPGHAN endoscopy and procedures committee. J Pediatr Gastroenterol Nutr. 2014 Sep;59(3):409-16.

2 Hunter A, Mamula P. Bowel preparation for pediatric colonoscopy procedures. J Pediatr Gastroenterol Nutr. 2010 Sep;51(3):254-61.

3 Dahshan A, Lin CH, Peters J, Thomas R, Tolia V. A randomized, prospective study to evaluate the efficacy and acceptance of three bowel preparations for colonoscopy in children. Am J Gastroenterol. 1999 Dec;94(12): 3497-501.

4 Pashankar DS, Uc A, Bishop WP. Polyethylene glycol 3350 without electrolytes: a new safe, effective, and palatable bowel preparation for colonoscopy in children. J Pediatr. 2004 Mar;144(3):358-62.

5 Phatak UP, Johnson S, Husain SZ, Pashankar DS. Two-day bowel preparation with polyethylene glycol 3350 and bisacodyl: a new, safe, and effective regimen for colonoscopy in children. J Pediatr Gastroenterol Nutr. 2011 Jul; 53(1):71-4

6 Elitsur R, Butcher L, Vicki L, Elitsur Y. Polyethylene glycol 3350 based colon cleaning protocol: $2 \mathrm{~d}$ vs $4 \mathrm{~d}$ head to head comparison. World J Gastrointest Endosc. 2013 Apr;5(4): 165-8.

7 Walia R, Steffen R, Feinberg L, Worley S, Mahajan L. Tolerability, safety, and efficacy of PEG 3350 as a 1-day bowel preparation in children. J Pediatr Gastroenterol Nutr. 2013 Feb;56(2):225-8.

8 Abbas MI, Nylund CM, Bruch CJ, Nazareno LG, Rogers PL. Prospective evaluation of 1-day polyethylene glycol-3350 bowel preparation regimen in children. J Pediatr Gastroenterol Nutr. 2013 Feb;56(2):220-4.

9 Sorser SA, Konanki V, Hursh A, Hagglund K, Lyons H. 1-day bowel preparation with polyethylene glycol 3350 is as effective and safe as a 3-day preparation for colonoscopy in children. BMC Res Notes. 2014 Sep;7(1):648.
10 Sahn B, Chen-Lim ML, Ciavardone D, Farace L, Jannelli F, Nieberle M, et al. Safety of a 1-Day Polyethylene Glycol 3350 Bowel Preparation for Colonoscopy in Children. J Pediatr Gastroenterol Nutr. 2016 Jul;63(1): 19-24.

11 Rostom A, Jolicoeur E. Validation of a new scale for the assessment of bowel preparation quality. Gastrointest Endosc. 2004 Apr;59(4): 482-6.

12 Lai EJ, Calderwood AH, Doros G, Fix OK, Jacobson BC. The Boston bowel preparation scale: a valid and reliable instrument for colonoscopy-oriented research. Gastrointest Endosc. 2009 Mar;69(3 Pt 2):620-5.

13 Di Nardo G, Aloi M, Cucchiara S, Spada C, Hassan C, Civitelli F, et al. Bowel preparations for colonoscopy: an RCT. Pediatrics. 2014 Aug;134(2):249-56.

14 Lane MM, Czyzewski DI, Chumpitazi BP Shulman RJ. Reliability and validity of a modified Bristol Stool Form Scale for children. J Pediatr. 2011 Sep;159(3):437-441.e1. 
15 Likert R. A technique for the measurement of attitudes. Arch Psychol. 1932;140:5-55.

16 Najafi M, Fallahi GH, Motamed F, Farahmand F, Khodadad A, Ghajarzadeh M, et al. Comparison of one and two-day bowel preparation with polyethylene glycol in pediatric colonoscopy. Turk J Gastroenterol. 2015 May;26(3):232-5.

17 Dehghani SM, Javaherizadeh H, Haghighat M, Imanieh MH, Ghanbari S. BOWEL PREPARATION BEFORE COLONOSCOPY FOR CHILDREN: comparison of efficacy of three different methods. Arq Gastroenterol. 2015 Dec;52(4):311-4.

18 Safder S, Demintieva Y, Rewalt M, Elitsur Y. Stool consistency and stool frequency are excellent clinical markers for adequate colon preparation after polyethylene glycol 3350 cleansing protocol: a prospective clinical study in children. Gastrointest Endosc. 2008 Dec;68(6):1131-5.

19 Jibaly R, LaChance J, Lecea NA, Ali N, Weber JE. The utility of PEG3350 without electrolytes for 2-day colonoscopy preparation in children. Eur J Pediatr Surg. 2011 Oct;21(5):318-21.

20 Terry NA, Chen-Lim ML, Ely E, Jatla M, Ciavardone D, Esch S, et al. Polyethylene glycol powder solution versus senna for bowel prep- aration for colonoscopy in children. J Pediatr Gastroenterol Nutr. 2013 Feb;56(2):215-9.

21 Lee JM, Lee JH, Kim ES, Lee JM, Yoo IK, Kim $\mathrm{SH}$, et al. The safety and effectiveness of 2-liter polyethylene glycol plus ascorbic acid in patients with liver cirrhosis: A retrospective observational study. Medicine (Baltimore). 2017 Dec;96(51):e9011.

22 Amato A, Radaelli F, Paggi S, Terruzzi V. Half doses of PEG-ES and senna vs. high-dose senna for bowel cleansing before colonoscopy: a randomized, investigator-blinded trial. Am J Gastroenterol. 2010 Mar;105(3):675-81.

23 Manukyan MN, Tolan K, Severge U, Attaallah W, Kebudi A, Cingi A. Prospective randomized comparison of oral sodium phosphate and sennoside $\mathrm{A}+\mathrm{B}$ calcium lavage for colonoscopy preparation. Surg Laparosc Endosc Percutan Tech. 2011 Apr;21(2):90-3.

24 Shavakhi A, Kianinia M, Torabi G, Nemati A, Saeidian B, Hoseinzadeh M, et al. High dose Senna or Poly Ethylene Glycol (PEG) for elective colonoscopy preparation: a prospective randomized investigator-blinded clinical trial. J Res Med Sci. 2011 Feb;16(2):149-55.

25 Yenidogan E, Okan I, Kayaoglu HA, Akgul GG, Sansal M, Tali S, et al. Same-day colonoscopy preparation with Senna alkaloids and bi- sacodyl tablets: a pilot study. World J Gastroenterol. 2014 Nov;20(41):15382-6.

26 Poyrazoglu OK, Yalniz M. Two low-dose bowel-cleansing regimens: efficacy and safety of senna and sodium phosphorus solution for colonoscopy. Patient Prefer Adherence. 2015 Sep;9:1325-31.

27 Adamiak T, Altaf M, Jensen MK, Sultan M, Ramprasad J, Ciecierega T, et al. One-day bowel preparation with polyethylene glycol3350: an effective regimen for colonoscopy in children. Gastrointest Endosc. 2010 Mar;71(3):573-7.

28 Ehrenpreis ED. Increased serum phosphate levels and calcium fluxes are seen in smaller individuals after a single dose of sodium phosphate colon cleansing solution: a pharmacokinetic analysis. Aliment Pharmacol Ther. 2009 Jun;29(11):1202-11.

29 US Food and Drug Administration. Information for healthcare professionals: oral sodium phosphate (OSP) products for bowel cleansing (marketed as Visicol and OsmoPrep, and oral sodium phosphate products available without a prescription). [Published 2014; Accessed: October 23, 2018]. Available from: http://www.fda.gov/drugs/drugsafety/postmarketdrugsafetyinformationforpatientsandproviders/ucm126084.htm. 